\title{
Adenosine kinase is a target for the prediction and prevention of epileptogenesis in mice
}

\author{
Tianfu Li, ${ }^{1}$ Gaoying Ren,, ${ }^{1}$ Theresa Lusardi, ${ }^{1}$ Andrew Wilz,, Jing Q. Lan, ${ }^{1}$ Takuji Iwasato, ${ }^{2}$ \\ Shigeyoshi Itohara, ${ }^{2}$ Roger P. Simon, ${ }^{1}$ and Detlev Boison ${ }^{1}$
}

${ }^{1}$ Robert S. Dow Neurobiology Laboratories, Legacy Clinical Research, Portland, Oregon, USA.

'Laboratory for Behavioral Genetics, RIKEN Brain Science Institute, Wako, Saitama, Japan.

\begin{abstract}
Astrogliosis is a pathological hallmark of the epileptic brain. The identification of mechanisms that link astrogliosis to neuronal dysfunction in epilepsy may provide new avenues for therapeutic intervention. Here we show that astrocyte-expressed adenosine kinase (ADK), a key negative regulator of the brain inhibitory molecule adenosine, is a potential predictor and modulator of epileptogenesis. In a mouse model of focal epileptogenesis, in which astrogliosis is restricted to the CA3 region of the hippocampus, we demonstrate that upregulation of ADK and spontaneous focal electroencephalographic seizures were both restricted to the affected CA3. Furthermore, spontaneous seizures in CA3 were mimicked in transgenic mice by overexpression of ADK in this brain region, implying that overexpression of ADK without astrogliosis is sufficient to cause seizures. Conversely, after pharmacological induction of an otherwise epileptogenesis-precipitating acute brain injury, transgenic mice with reduced forebrain ADK were resistant to subsequent epileptogenesis. Likewise, ADK-deficient ES cell-derived brain implants suppressed astrogliosis, upregulation of ADK, and spontaneous seizures in WT mice when implanted after the epileptogenesis-precipitating brain injury. Our findings suggest that astrocyte-based ADK provides a critical link between astrogliosis and neuronal dysfunction in epilepsy.
\end{abstract}

\section{Introduction}

Current epilepsy therapies largely rely on the suppression of spontaneous seizures by pharmacotherapy or surgical intervention, but to date no effective prophylaxis or true pharmacotherapeutic cure is available. Epileptogenesis, i.e., the process that leads to epilepsy and spontaneous seizures, is thought to be triggered by an initial acute brain injury (e.g., status epilepticus [SE]), followed by progressive neuronal cell loss, mossy fiber sprouting, and formation of an astrogliotic scar (1). However, it is presently unclear why some brain injuries evolve into epilepsy while others do not. Therefore, the identification of diagnostic markers to predict epileptogenesis is of utmost importance. The identification of astrogliosis as a hallmark in brain of epileptogenesis and the identification of astrocytes as important modulators of neuronal activity imply that dysfunction of astrocytes might play a key role in the pathogenesis of epilepsy $(2,3)$.

Animal models of epileptogenesis have been developed that closely mimic the pathogenesis of human mesial temporal lobe epilepsy, a form of human focal epilepsy that is frequently associated with progression to chronic intractable epilepsy. These models rely on the intrahippocampal (4), intraamygdaloid (5), or intracerebroventricular (6) administration of small doses of kainic acid (KA). As a primary consequence of KA injection, SE is elicited, which in turn - during a time span of several weeks - leads to a characteristic pattern of hippocampal cell death, mossy fiber sprouting, astrogliosis, and spontaneous recurrent seizures (4-6).

Adenosine is an inhibitory modulator of brain activity. It acts at 4 G-protein-coupled receptor subtypes $\left(A_{1}, A_{2 A}, A_{2 B}\right.$, and $\left.A_{3}\right)(7)$

Nonstandard abbreviations used: ADK, adenosine kinase; KA, kainic acid; NP, neural precursor cell; SE, status epilepticus.

Conflict of interest: The authors have declared that no conflict of interest exists. Citation for this article: J. Clin. Invest. 118:571-582 (2008). doi:10.1172/JCI33737. and in hippocampus exerts predominantly inhibitory functions by activation of $A_{1}$ receptors $\left(A_{1} R s\right)(8)$. The inhibitory actions of adenosine can be used therapeutically to suppress seizures (9) and are considered important for maintaining postictal depression (10) and for restoring the metabolic equilibrium following seizures (11). However, despite more than 20 years of research on the role of adenosine in experimentally induced seizures and the identification of adenosine as endogenous anticonvulsant of the brain (12-15), the pathogenetic role of the adenosine system in epileptogenesis remains understudied.

Ambient concentrations of adenosine are largely regulated by the activity of its major metabolic enzyme adenosine kinase (ADK) (16), which in the adult brain is predominantly expressed in astrocytes (17). Consequently, astrogliosis in epilepsy is associated with upregulation of ADK (18). Transgenic upregulation of ADK in brain leads to a reduction in the tone of ambient adenosine and therefore is associated with increased susceptibility to seizures (19) and ischemic cell death (20). These findings provide a neurochemical rationale for therapeutic intervention (21). Consequently, cultured cells engineered to release adenosine by disrupting their Adk genes were tested as therapeutic brain implants in the rat kindling model (22). These studies have provided the proof-of-concept that focal augmentation of adenosine by cellular brain implants can reduce induced (kindled) seizures and the progression of kindled seizure severity $(22,23)$. However, it remains to be demonstrated whether focal augmentation of adenosine can prevent the development of spontaneous seizures, i.e., true epileptogenesis.

To characterize ADK as a target for the prediction and prevention of epileptogenesis we followed 3 independent lines of research: characterization of the pathogenetic role of ADK in a mouse model of focal epileptogenesis, study of epileptogenesis in a panel of transgenic mice with altered brain expression levels of ADK, and intracerebral transplantation of ADK-deficient stem cells to 
contralateral
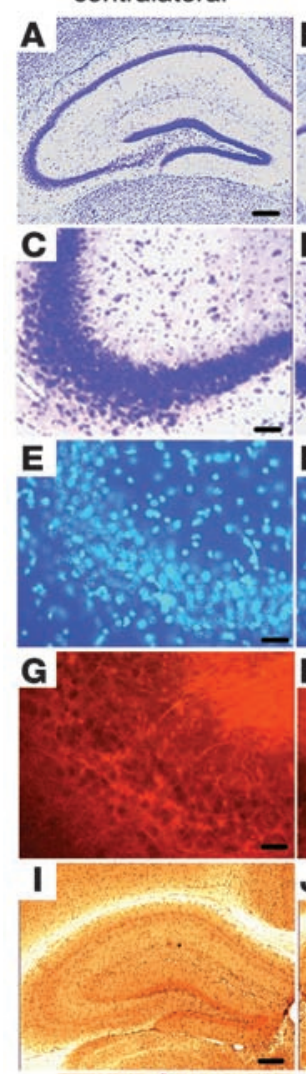

K

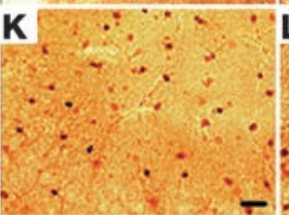

M

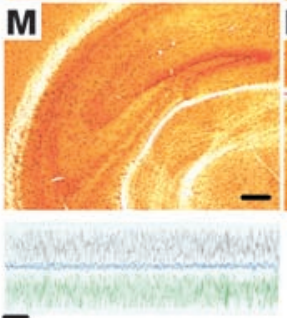

CA3 ipsilateral

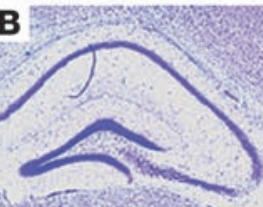

D.t.
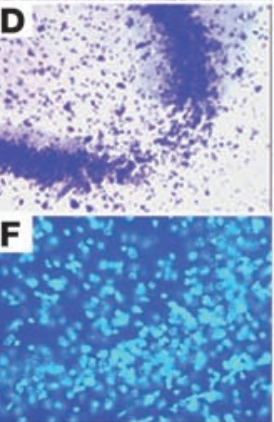

H
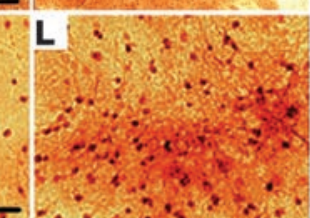

N.
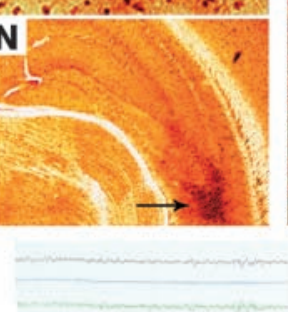

CA1
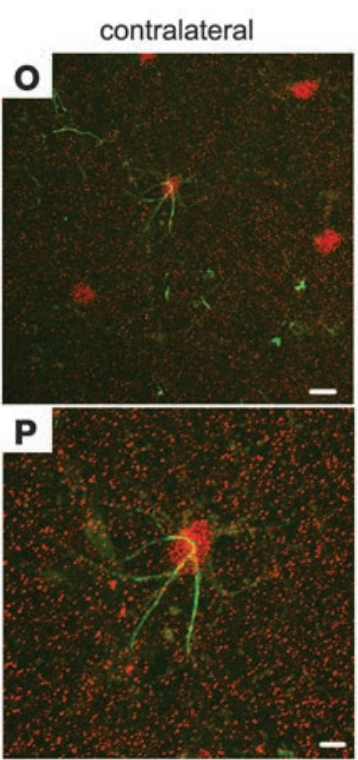

ipsilateral
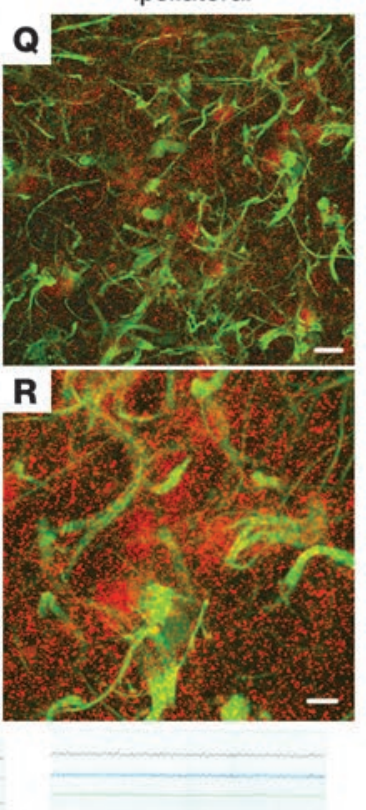

Cortex

\section{Figure 1}

Characterization of spontaneously epileptic mice 3 weeks after intraamygdaloid injection of KA. (A-D) Representative cresyl violet-stained coronal brain section showing the hippocampal formation contralateral (A and $\mathbf{C})$ or ipsilateral ( $\mathbf{B}$ and $\mathbf{D}$ ) to the KA injection. Note the CA3 selective cell loss in the ipsilateral hippocampus ( $\mathbf{B}$ and $\mathbf{D})$. ( $\mathbf{E}$ and $\mathbf{F}$ ) Adjacent coronal brain section from the same animal stained with the nuclear stain DAPI showing an increase in the number of cell nuclei in the injured CA3. ( $\mathbf{G}$ and $\mathbf{H}$ ) The same section stained with NFM indicating the presence of dense neuronal networks within the injured CA3. (I-N) ADK immunoreactivity within the hippocampal formation visualized with diaminobenzidine contralateral $(\mathbf{I}, \mathbf{K}$, and $\mathbf{M})$ or ipsilateral $(\mathbf{J}, \mathbf{L}$, and $\mathbf{N})$ to the KA injection. Note the focal overexpression of ADK in the ipsilateral CA3. I-L are from the same animal and adjacent to those shown in $\mathbf{A}-\mathbf{H}$. $\mathbf{M}$ and $\mathbf{N}$ are derived from the same animal at a more caudal location, representing the level of cell transplantations (see Figure 10). Arrows in $\mathbf{J}$ and $\mathbf{N}$ point to upregulated ADK in ipsilateral CA3. (0-R) Confocal analysis of ADK (red) and GFAP (green) immunoreactivity in the contralateral ( $\mathbf{O}$ and $\mathbf{P})$ and ipsilateral $(\mathbf{Q}$ and $\mathbf{R}) \mathrm{CA3}$. Note prominent astrogliosis (GFAP) and overexpression and redistribution of $A D K$ in the ipsilateral $C A 3(\mathbf{Q}$ and $\mathbf{R})$. Bottom: Representative EEG recordings obtained from electrodes inserted into the ipsilateral CA3 or CA1 or placed onto the cortex. Scale bars: $300 \mu \mathrm{m}(\mathbf{A}, \mathbf{B}, \mathbf{I}, \mathbf{J}, \mathbf{M}$, and N), $75 \mu \mathrm{m}(\mathbf{C}$ and $\mathbf{D}), 37.5 \mu \mathrm{m}$ (E, F, G, H, K, and $\mathbf{L}), 12 \mu \mathrm{m}(\mathbf{O}$ and $\mathbf{Q})$, and $5 \mu \mathrm{m}$ ( $\mathbf{P}$ and $\mathbf{R})$. EEG scale bar: $2 \mathrm{~s}$. modify epileptogenesis. Our results support the ADK hypothesis of epileptogenesis and define ADK as a promising target for the prediction and prevention of epileptogenesis.

\section{Results}

Astrogliosis, upregulation of $A D K$, and focal spontaneous seizures characterize epileptogenesis in WT mice. Adult male WT C57BL/6 mice $(n=6)$ received intraamygdaloid KA injections. As a consequence of the KA injection, all animals developed nonconvulsive electrographic SE and CA3-selective neuronal cell loss, as previously described (24). All animals survived a time span of 3 weeks, when electrodes were implanted into the ipsilateral CA3 region. During 8 hours of continuous monitoring, spontaneous focal seizures were recorded from the CA3 region of all animals, with $4.3 \pm 1.5$ seizures per hour and an average duration of $17.5 \pm 5.8$ seconds (Figure 1, bottom left), indicating epileptogenesis. It is important to note that (a) spontaneous seizures were observed in every KA-injected animal, (b) that electrographic seizures were never associated with convulsions, and (c) that seizure activity was specific to the injured ipsilateral CA3 region. In additional animals ( $n=6$ each) with electrodes placed in CA1 or onto the cortex, we were not able to record any seizures (Figure 1), demonstrating that epileptogenesis in WT animals is highly specific for the ipsilateral CA3 region, the region of acute neuronal cell loss. In a separate set of animals $(n=3)$ that were recorded daily after SE using CA3 electrodes, the first onset of spontaneous seizures was determined. In all animals, spontaneous CA3 seizures first emerged 12 days after SE $(3.1 \pm 0.9$ seizures per hour, with a duration of $15.7 \pm 6.0$ seconds). 
A

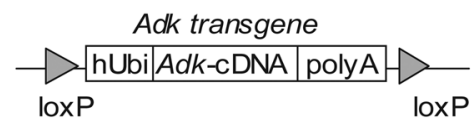

B

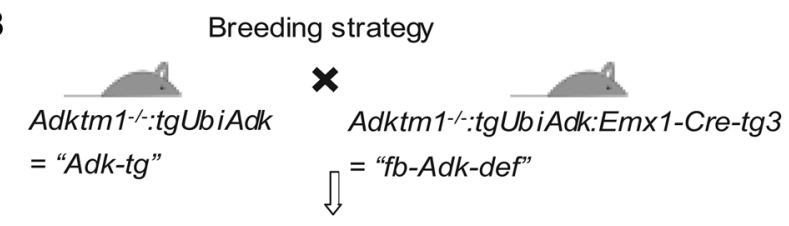

$50 \%$ fb-Adk-def

$50 \%$ Adk-tg

C

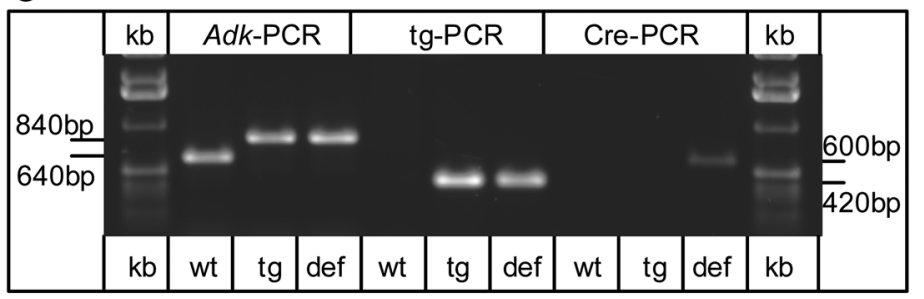

D
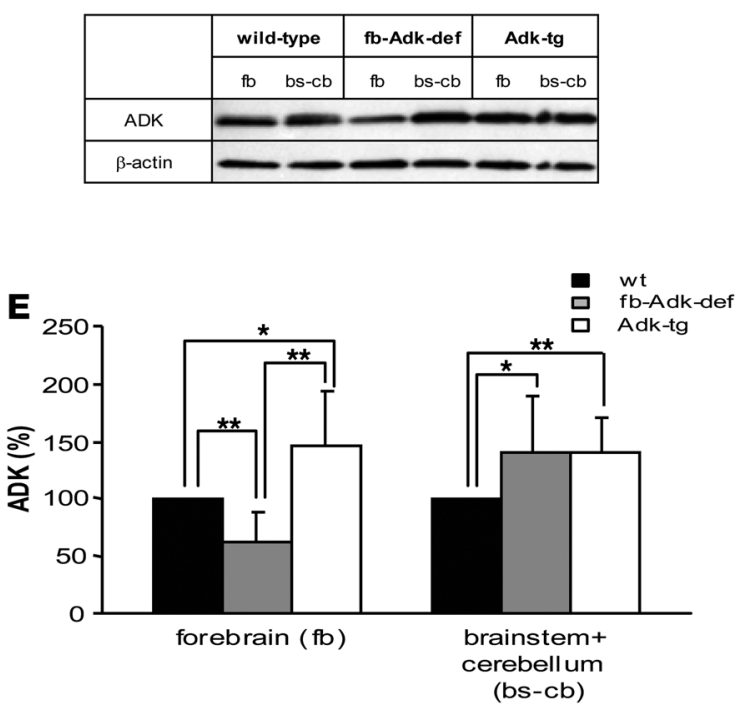

Figure 2

Generation and characterization of $f b$-Adk-def mice. (A) The short isoform of mouse Adk cDNA is located between a human ubiquitin promoter (hUbi) and an SV40 polyA sequence. The UbiAdk transgene is flanked by loxP sites. (B) Adk-Tg mice, which were homozygous for the Adk-KO and the TgUbiAdk transgene, were bred with $f b$-Adk-def mice, which were heterozygous for Cre. From these crosses, Adk-Tg and $f b$-Adk-def littermates were produced. (C) Representative PCR of selected animals. Tg, Adk-Tg; def, fb-Adk-def. DNA was amplified with a PCR specific for either the WT $(640 \mathrm{bp})$ or the KO allele (840 bp) of the endogenous Adk gene (Adk-PCR), a PCR specific for the Adk transgene (Tg-PCR), giving rise to a 420-bp amplification product, or a PCR specific for the Cre gene (Cre-PCR), giving rise to a 600-bp product. (D) Representative western blot from adult WT, fb-Adk-def, and Adk-Tg mice. Top: ADK-immunoreactive bands from forebrain (fb) or brainstem plus cerebellum (bs-cb). Bottom: $\beta$-Actin-immunoreactive bands were used to normalize for equal loading. (E) Quantitative analysis of ADK levels based on 3 western blots performed with samples from $n=2$ animals from each genotype. ADK levels were first normalized to equal loading according to the $\beta$-actin standard. These values were then normalized to the respective WT samples (set as $100 \%)$. Data represent the mean \pm SD of 6 samples. ${ }^{\star} P<0.05 ;{ }^{*} P<0.01$, paired comparisons in $t$ test.

After completion of EEG recordings, all animals were sacrificed for histology. Analysis of cresyl violet-stained brain sections showed ipsilateral CA3 injury (Figure 1, B and D), indicating the presence of the epileptogenesis-precipitating acute injury, while the contralateral CA3 region was spared from injury (Figure 1, $A$ and $C$ ). Fluorescence staining for nuclei revealed the presence of an increased number of nuclei in the ipsilateral compared with the contralateral CA3 (Figure 1, E and F), indicating an increase in nonneuronal cells within the injured CA3. To investigate whether neuronal cell loss in the injured CA3 (Figure 1D) was accompanied by a loss of neuronal processes, we performed an immunofluorescence staining with a marker for neuronal filaments (NFM) (Figure 1, G and H). Despite the reduction of neuronal cell bodies within the injured CA3, the amount of NFM immunoreactivity was unchanged, indicating a dense network of neuronal processes within the injured CA3 likely originating from neuronal cell bodies elsewhere. Since astrogliosis and overexpression of endogenous ADK may be linked to epileptogenesis, we analyzed expression of the astrocyte markers GFAP and ADK in the hippocampal formation in more detail. All animals displayed a striking and highly localized astrogliotic response covering the rostrocaudal extent of the ipsilateral CA3 (Figure 1, J, L, and N), with colocalized overexpression of GFAP and ADK (Figure 1, Q and $\mathrm{R}$ ), while the contralateral hippocampus was spared from astrogliosis and upregulation of ADK (Figure 1, I, K, M, O, and $\mathrm{P})$. Confocal imaging (Figure $1, \mathrm{O}, \mathrm{P}, \mathrm{Q}$, and $\mathrm{R}$ ) revealed not only increased $\mathrm{ADK}$ immunoreactivity and the predominantly nuclear localization of the enzyme but also a redistribution and spread of ADK immunoreactivity to extranuclear locations (Figure 1R).

Generation of transgenic mice with altered levels of brain $A D K$. To provide experimental evidence that upregulation of ADK during epileptogenesis is causative for the development of spontaneous seizures in epileptogenesis, we created a panel of transgenic mice with either enhanced or reduced expression of brain ADK. To generate a mouse model with a forebrain-selective reduction of ADK, we bred Emx1-Cre-Tg3 mice, which express Cre-recombinase in neurons and astrocytes of the telencephalon (25), with ADK transgenic mice, which carry a loxP-flanked ADK transgene (Adktm1 $1^{-1-}:$ TgUbiAdk) (19) (Figure 2A) in an otherwise lethal ADK KO (Adktm1-/-) background (26). Adktm1 1- $: \operatorname{Tg}(U b i A d k)$ (referred to here as "Adk-Tg") and Adktm1 1--:Tg(UbiAdk):Emx1-Cre-Tg3 (referred to here as "fb-Adk-def") offspring were produced as littermates (Figure 2B). Genotyping was performed at weaning and again retrospectively from all experimental animals (Figure 2C).

To demonstrate that Cre recombination of the ADK transgene in $f b$-Adk-def mice resulted in a forebrain-selective reduction of ADK, the expression pattern of ADK was analyzed by immunoperoxidase staining on coronal sections from adult WT, $f b-A d k-d e f$, and $A d k-T g$ mice ( $n=3$ per group). In WT sections, strong ADK immunoreactivity was largely restricted to nuclei and cell bodies of evenly distributed astrocytes (Figure 3, A and B), as previously described (17), whereas pyramidal cells of the CA3 region (Figure 3B) lacked specific ADK immunoreactivity. In contrast, $A d k-T g$ mice were characterized by lack of the endogenous astrocytic ADK (lack of 


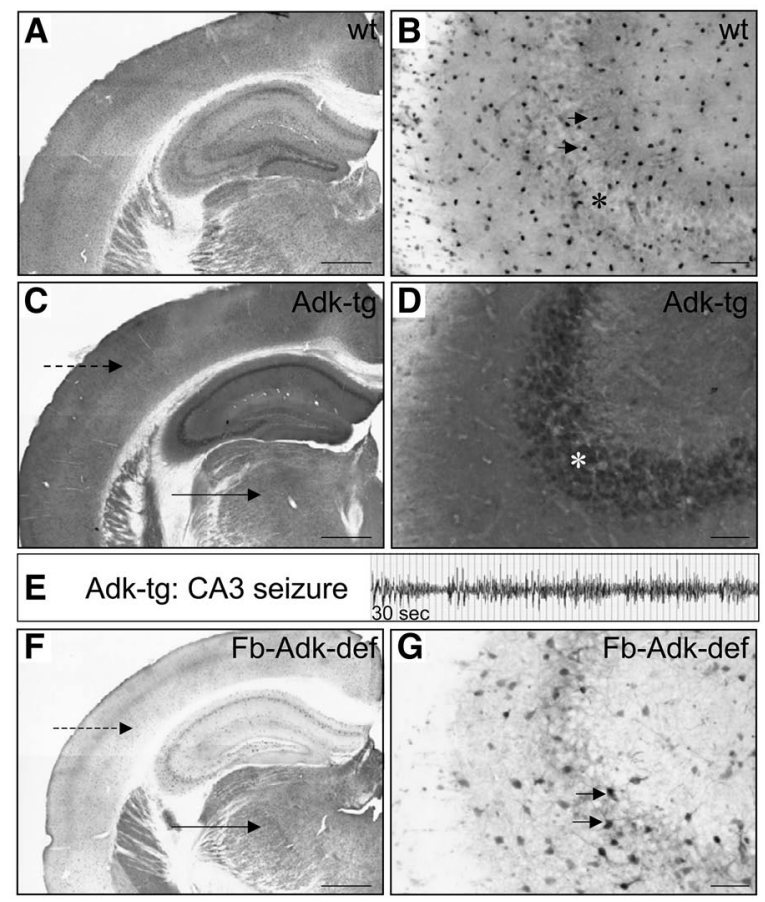

discrete staining of astrocytic nuclei and cell bodies due to genetic disruption of the endogenous Adk gene), which was replaced by a ubiquitous overexpression of transgenic ADK (Figure 3C), resulting in strong diffuse immunoreactivity in neurons and astrocytes all over the brain but lack of nuclear localization. Most importantly, a novel neuronal expression of ADK was found, particularly in pyramidal neurons of the CA3 region (Figure 3D). In $f b$-Adk-def mice, ADK reduction - induced by Cre-mediated deletion of the loxP-flanked transgene - became apparent in the whole dorsal telencephalon (Figure 3F), encompassing cerebral cortex and hippocampus, while basal brain regions continued to display the diffuse overexpression of ADK, in line with unaltered expression of the Adk transgene (Figure 3, C and F). Only a few individual cells with neuronal morphology within the forebrain remained positive for ADK (Figure 3G), most likely GABAergic interneurons, a cell type in which Emx1-Cre is not expressed (25).

To quantify the reduction of ADK in the dorsal telencephalon of $f b$-Adk-def mice, a western blot analysis was performed comparing the intensity of ADK immunoreactivity in dorsal telencephalon with brain stem plus cerebellum from tissue samples taken from WT, $f b$-Adk-def, and $A d k$ - $T g$ mice (Figure 2D). The forebrains of $f b$-Adk-def mice had significantly reduced levels of ADK, with an average of $62 \% \pm 27 \%(P<0.01)$ compared with WT forebrain $(100 \%)$, while ADK in forebrain of Adk-Tg mice was elevated to $147 \% \pm 49 \%$ of WT levels $(P<0.05)$. In both $f b$-Adk-def and $A d k-T g$ mice, brainstem plus cerebellum ADK levels were elevated to an average of $141 \% \pm 50 \%$ and $141 \% \pm 30 \%$ of WT levels, respectively (Figure $2 \mathrm{E}$ ).

Adk-Tg mice are characterized by spontaneous seizures in CA3 and lethal $K A$-induced SE. If upregulation of $\mathrm{ADK}$ in the $\mathrm{CA} 3$ region during epileptogenesis (Figure 1) is the cause for seizures rather than astrogliosis, then transgenic overexpression of ADK within the CA3 should result in spontaneous seizures. Adk-Tg mice display a particularly prominent overexpression of ADK within the CA3 region (Figure $3 \mathrm{D})$. To test for spontaneous electrographic seizures, we therefore

\section{Figure 3}

Photomicrographs of ADK immunoreactivity in coronal brain sections of adult mice, processed for immunoperoxidase staining under identical conditions. (A) Hippocampal formation of a WT brain showing a homogenous distribution of individual cells expressing ADK. (B) CA3 region (asterisk) of WT mouse at higher magnification showing the restriction of ADK expression to individual astrocytes (arrows). (C) Hippocampal formation of an Adk-Tg brain showing ubiquitous overexpression of transgenic ADK coupled with a loss of punctate staining of endogenous ADK in individual cells. Note the strong ADK immunoreactivity in pyramidal cell neurons. Dashed arrow, cortex; solid arrow, thalamus. (D) CA3 region of an Adk- Tg mouse at higher magnification showing overexpression of ADK in CA3 neurons (asterisk). (E) Spontaneous seizure recorded from the CA3 region of an Adk-Tg mouse. Trace represents $30 \mathrm{~s}$ of recording time. (F) Hippocampal formation of an $\mathrm{fb}$-Adk-def brain showing reduced ADK staining in hippocampus and cortex (dashed arrow) compared with thalamus (solid arrow) and striatum of the same slice and compared with both WT and Adk-Tg hippocampus. (G) CA3 region of an $f b$-Adk-def mouse at higher magnification showing global reduction of $A D K$ but individual remaining ADK-positive cells (arrows). Scale bars: $500 \mu \mathrm{m}$ (A, C, and F), $50 \mu \mathrm{m}$ (B, D, and G). inserted bipolar recording electrodes directly into the CA3 region of $A d k-T g$ mice $(n=4)$. Eight hours of continuous EEG recordings yielded frequent spontaneous seizures (Figure 3E), with an average of $4.8 \pm 1.5$ seizures per hour, each seizure lasting $26.7 \pm 13.2$ seconds. Remarkably, transgenic overexpression of ADK yielded a similar seizure frequency as astrogliotic upregulation of ADK in epileptic WT mice ( $4.3 \pm 1.5$ seizures per hour).

To determine the susceptibility to seizures, $\operatorname{Adk}-\operatorname{Tg}(n=5)$ and WT $(n=6)$ mice received intraamygdaloid injections of KA. Following the KA injections, seizure activity was monitored in EEG recordings with a set of 3 cortical electrodes. After 30 minutes, seizures were terminated with lorazepam. Seizures were graded into type I-IV activities, as previously described (24). While WT mice developed typical type IV seizures (Figure 4), Adk-Tg mice developed type IV seizures of increased intensity (Figure 4). In $A d k-T g$ mice, the duration of type IV activity $(713 \pm 186 \mathrm{~s})$ was significantly

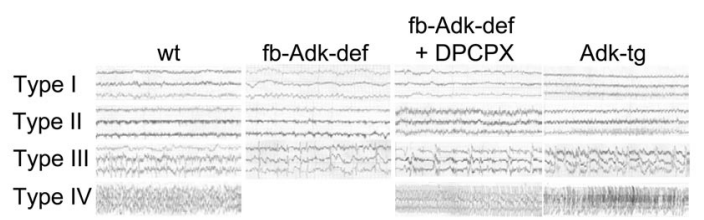

\section{Figure 4}

Electrographic seizure patterns recorded after intraamygdaloid injection of KA. Representative traces show type I (baseline), type II (ictal fast activity), type III (high-voltage spikes of $<1 \mathrm{~Hz}$ frequency superimposed over type II activity), and type IV (high-voltage, polyspike paroxysmal discharges of $>1 \mathrm{~Hz}$ frequency) electrographic activity recorded from 3 cortical electrodes after the injection of $0.3 \mu \mathrm{g} \mathrm{KA}$ into the basolateral amygdala of WT mice, $f b$-Adk-def mice, $f b-A d k$-def mice pretreated with $1 \mathrm{mg} / \mathrm{kg}$ DPCPX, and Adk-Tg mice. Note the aggravation of type IV seizures in Adk-Tg mice and the lack of type IV seizures in $f b$-Adk-def mice. Each trace represents $20 \mathrm{~s}$ of recording time. 
Table 1

Mortality, electrographic seizure activity, and cell death 24 hours after intraamygdaloid KA injection

$\begin{array}{lcccc}\text { Parameter } & \text { WT } & \text { Adk-Tg } & \text { fb-Adk-def } & \text { fb-Adk-def+ DPCPX } \\ \text { Mortality }(24 \mathrm{~h}) & 0 / 6 & 2 / 5 & 0 / 5 & 0 / 5 \\ \text { Mortality (3 d) } & 0 / 5 & 5 / 5 & 0 / 5 & 0 / 5 \\ \text { Average type IV seizure duration (s) } & 443.3 \pm 147.2(n=6) & 713.3 \pm 185.8^{A}(n=3) & 0(n=5) & 318.0 \pm 100.6(n=5) \\ \text { TUNEL count, ipsilateral CA3 } & 73.8 \pm 17.1 & 117.6 \pm 31.1^{A} & 0 & 51.8 \pm 17.7 \\ \text { TUNEL count, ipsilateral CA1B } & 0 & 38.6 \pm 4.0^{\mathrm{C}} & 0 & 0 \\ \text { TUNEL count, contralateral CA3B } & 0 & 28.3 \pm 10.4^{\mathrm{D}} & 0 & 0 \\ \text { TUNEL count, contralateral CA1B } & 0 & 25.0 \pm 5.6^{\mathrm{D}} & 0 & 0\end{array}$

AP $<0.05$ compared with WT by $t$ test. BThe average number of TUNEL-positive cells per brain section was determined by counting TUNEL-positive cells in 3 brain sections from $n=3$ mice per genotype. ${ }^{C} P<0.001$; $D P<0.01$ compared with WT by $t$ test.

prolonged $(P<0.05)$ compared with WT (Table 1$)$ and finally led to the death of 2 of 5 animals within 24 hours (Table 1 ). In an additional group of KA-injected $A d k-T g$ mice $(n=5)$ we observed to investigate long-term consequences of KA-induced SE, all animals died within 3 days of the KA injection (Table 1).

To investigate the influence of overexpressed ADK on the development of acute seizure-induced hippocampal injury, 24 hours after KA injection all remaining $A d k-T g$ mice $(n=3)$ were analyzed histologically by cresyl violet and TUNEL staining (Figure 5 and Table 1). KA-injected WT mice developed a well-defined lesion in the ipsilateral CA3 of the hippocampus, which was characterized by prominent TUNEL-positive cells, being indicative of ongoing cell death (Figure 5, B and C). KA-induced seizures affected neither the contralateral hippocampus (Figure 5A) nor any other brain region. In contrast, $\mathrm{ADK}$ overexpression in $A d k-T g$ mice led to highly aggravated cell loss in the ipsilateral CA3 region (Figure $5, \mathrm{~K}$ and $\mathrm{L}$, and Table 1 ) as well as to an extension of cell loss in the ipsilateral CA1 region (Figure $5 \mathrm{~N}$ ) and dentate gyrus (Figure $5 \mathrm{~K}$ ), and most remarkably, to significant cell loss in the contralateral hippocampus (Figure 5, J, M, and O, and Table 1).

fb-Adk-def mice exhibit reduced $K A$-induced $S E$ and are protected from acute brain injury. To determine whether seizure susceptibility is reduced under conditions of reduced ADK, $f b$-Adk-def mice $(n=5)$ received intraamygdaloid injections of KA. An additional group of $f b$-Adk-def mice $(n=5)$ received an i.p. injection of the adenosine $\mathrm{A}_{1} \mathrm{R}$ antagonist DPCPX $(1 \mathrm{mg} / \mathrm{kg}) 30$ minutes prior to KA. Following the KA injections, seizure activity was monitored in EEG recordings as described above. In contrast to WT mice, type IV

\section{Figure 5}

Epilepsy-associated cell loss. Representative micrographs of the hippocampal formation from coronal brain sections taken from WT mice (A-C), fb-Adk-def mice (D-F), fb-Adk-def mice pretreated with $1 \mathrm{mg} / \mathrm{kg}$ i.p. DPCPX (G-I), and $A d k-T g$ mice (J-O) $24 \mathrm{~h}$ after intraamygdaloid injection of KA. Sections were stained either with cresyl violet or with TUNEL (green). (A-C) Typical cell death in the CA3 region of the hippocampus of WT mice ipsilateral (ipsi) to the KA-injected amygdala. (D-F) fb-Adk-def mice did not display any signs of cell loss. (G-I) After pretreatment with DPCPX, fb-Adk-def mice developed cell loss, which was comparable with WT animals. (J-O) Adk-Tg mice showed aggravated cell loss in the ipsilateral CA3 region and novel cell loss in the ipsilateral $\mathrm{CA} 1$ region $(\mathbf{M})$, and the contralateral $\mathrm{CA} 1$ (N) and CA3 (O) regions. Scale bars: $300 \mu \mathrm{m}(\mathbf{A}, \mathbf{B}, \mathbf{D}, \mathbf{E}, \mathbf{G}, \mathbf{H}$, $\mathbf{J}$, and $\mathbf{K}) ; 75 \mu \mathrm{m}(\mathbf{C}, \mathbf{F}, \mathbf{I}, \mathbf{L}, \mathbf{M}, \mathbf{N}$, and $\mathbf{O})$.
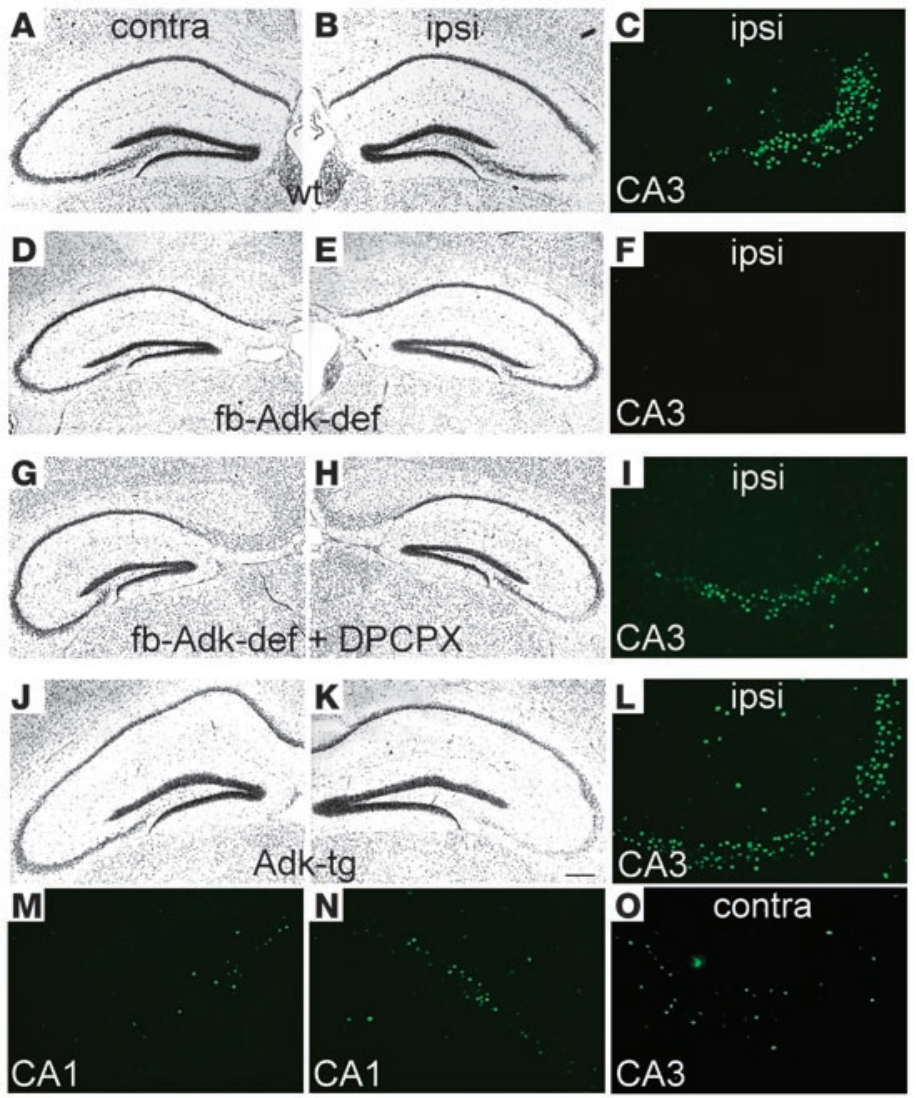
A

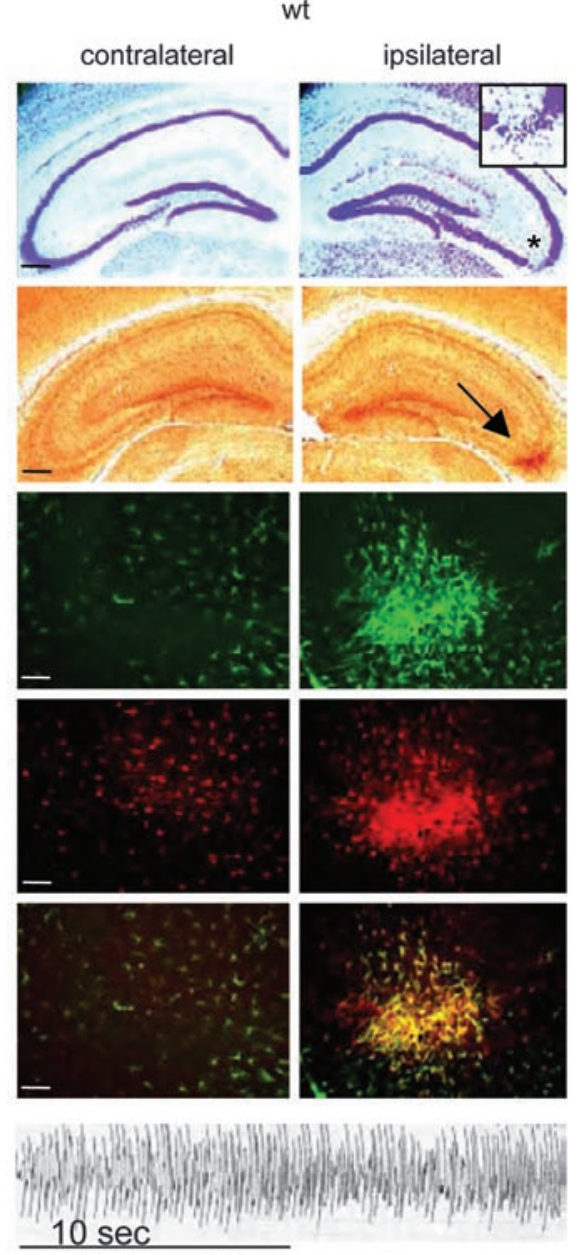

B

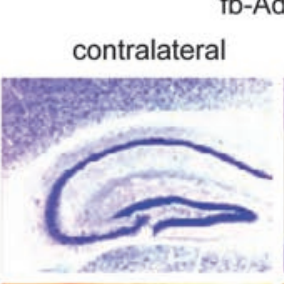

fb-Adk-def
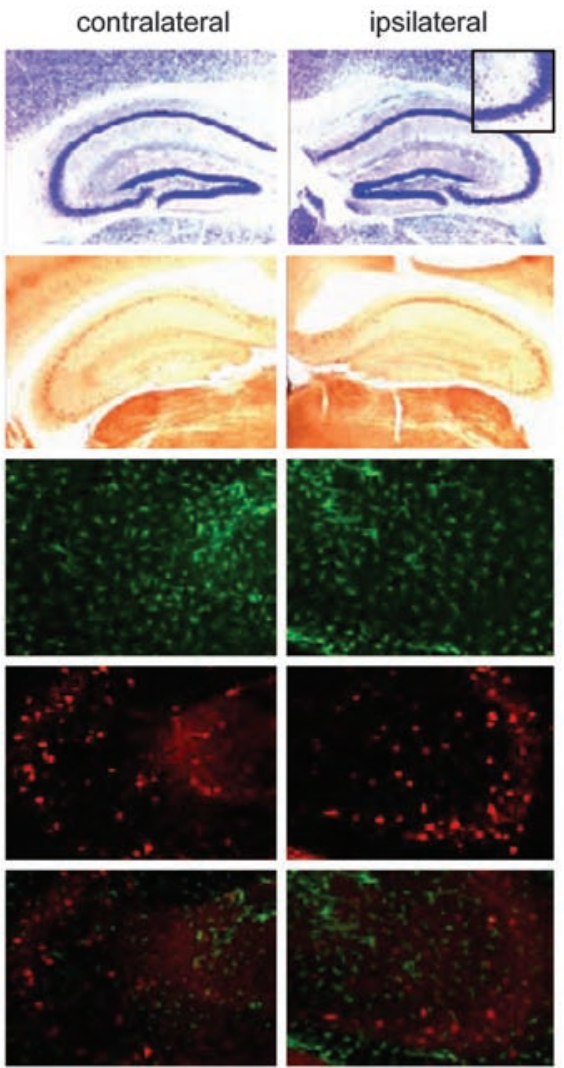

C

fb-Adk-def

+ DPCPX

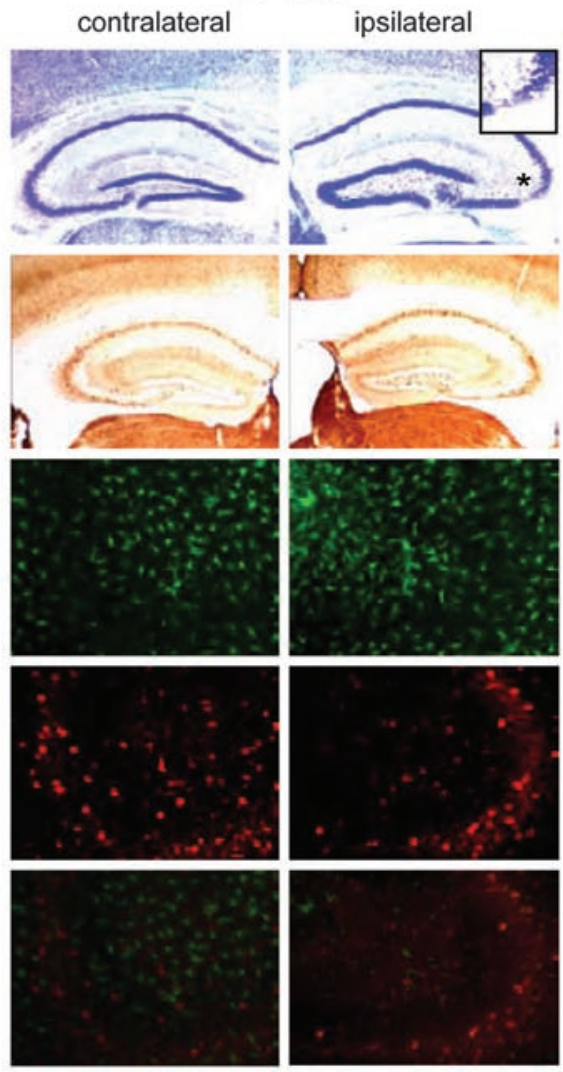

spontaneous CA3 seizure

\section{Figure 6}

Assessment of epileptogenesis 3 weeks after KA/SE. Representative micrographs and EEGs taken from the hippocampal formation of WT mice (A), $f b-A d k$-def mice (B), and fb-Adk-def mice pretreated with $1 \mathrm{mg} / \mathrm{kg}$ i.p. DPCPX (C) 3 weeks after intraamygdaloid injection of KA. First row: Cresyl violet-stained sections showing the characteristic ipsilateral KA/SE-induced CA3 lesion in WT and fb-Adk-def/DPCPX mice (A and C, asterisks), but not in fb-Adk-def mice. Insets show enlarged ipsilateral CA3 (magnification, $\times 2.25$ ). Second row: ADK immunoreactivity visualized with DAB. Note that ADK is exclusively upregulated in the ispsilateral CA3 of WT animals, corresponding to the location of the primary brain injury (arrow). Third row: GFAP immunofluorescence staining showing astrogliosis in the ipsilateral CA3 of WT mice but lack of astrogliosis in $f b-A d k-d e f$ and $f b-A d k-d e f / D P C P X$ mice. Fourth row: ADK immunofluorescence staining of the same specimen. Fifth row: Overlay of GFAP (green) and ADK (red) immunofluorescence showing colocalization (yellow) of GFAP and ADK in the astrogliotic CA3 region of WT mice. Sixth row: Representative intrahippocampal EEG recordings taken from CA3 recordings showing spontaneous focal seizures in WT animals, while $f b-A d k$-def and $f b-A d k$ - $d e f / D P C P X$ mice were protected from the development of spontaneous seizures. Scale bars: $300 \mu \mathrm{m}$ (black bars); $75 \mu \mathrm{m}$ (white bars). EEG scale bar: $10 \mathrm{~s}$.

seizures were never seen in $f b$-Adk-def mice (Figure 4). These mice developed normal types I and II activity, and type III activity of reduced intensity. This strong protection from seizures was due to elevated adenosine in the forebrain of the mutants acting via the $A_{1} R$, since application of the $A_{1} R$ antagonist DPCPX completely abolished the protection, resulting in type IV seizure activity (Figure 4$)$ and duration (318 \pm 101 seconds), which was comparable to WT (443 \pm 147 seconds) $(P>0.05$; Table 1$)$.

To investigate the influence of reduced levels of ADK on the development of acute seizure-induced hippocampal injury, 24 hours after KA injection all animals were analyzed histologically by cresyl violet and TUNEL staining (Figure 5 and Table 1). In contrast to WT control animals, $f b$-Adk-def mice were completely protected from any injury (Figure 5, D-F). In $f b$-Adk-def mice, not a single TUNEL-positive cell was detected (Figure $5 \mathrm{~F}$ and Table 1), indicating a powerful neuroprotective effect as a consequence of ADK deficiency. The obvious neuroprotection in $f b$-Adk-def mice was reversed when animals were pretreated with DPCPX, including hippocampal lesions (Figure 5, G-I) reminiscent of the lesions seen in WT animals and a similar number of TUNEL-positive cells per section (Table 1). To demonstrate that the chosen dose of DPCPX $(1 \mathrm{mg} / \mathrm{kg}$, i.p.) acts more by blocking effects of increased adenosine (as expected in forebrains of $f b$-Adk-def mice) on the $\mathrm{A}_{1} \mathrm{R}$ rather then interfering with "normal" levels of adenosine in WT mice, an additional group of WT mice $(n=6)$ was injected with DPCPX 30 minutes prior to intraamygdaloid KA injection. Brains of these 


\section{CA3-EEGs}

A

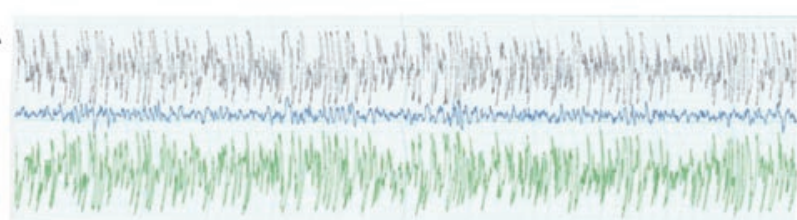

B

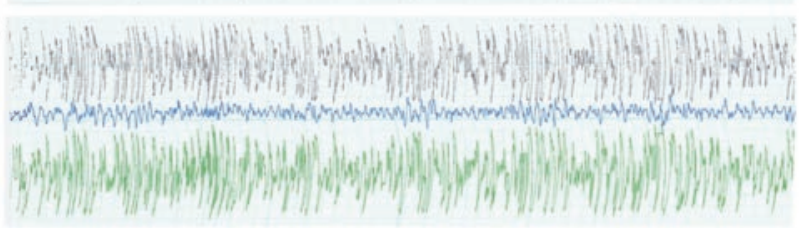

C

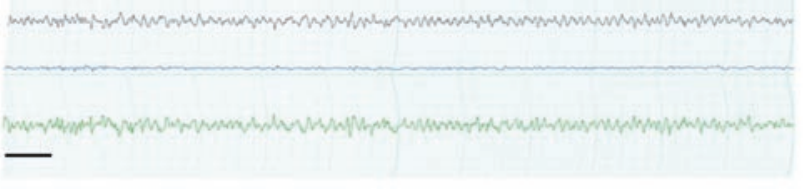

animals were analyzed 24 hours later for the presence of TUNELpositive cells in the CA3. We found 78.2 \pm 20.3 TUNEL-positive cells per CA3 section, a value comparable $(P>0.05)$ with KA-injected WT mice not treated with DPCPX $(73.8 \pm 17.1$; Table 1$)$, indicating that the chosen dose of DPCPX normalized the effects of increased adenosine on $\mathrm{A}_{1} \mathrm{Rs}$ in $f b$-Adk-def mice and therefore recreates a WTlike acute injury in these mutant animals.

$f b$-Adk-def mice are resistant to epileptogenesis. To demonstrate that ADK could, via adenosine, not only have these acute effects but also influence epileptogenesis chronically, we compared consequences of KA injection after 3 weeks in WT mice (acute KA-induced SE and CA3 cell loss), fb-Adk-def mice (no acute changes), and fb-Adk-def mice pretreated 30 minutes before KA injection with a single dose of DPCPX (restoration of acute WT-like SE and CA3 cell death; Figures 4 and 5). Due to the transient nature of $A_{1}$ R blockade, DPCPXpretreated $f b$-Adk-def mice $(f b$-Adk-def/DPCPX) developed an acute epileptogenesis-initiating injury (SE, CA3 cell loss), but thereafter were under the influence of elevated forebrain adenosine.

WT, $f b-A d k-d e f$, and $f b-A d k-d e f / D P C P X$ mice $(n=5$ per group) received intraamygdaloid KA injections as described above. As a consequence of KA injection, all animals except $f b$-Adk-def mice developed SE analogous to that shown in Figure 4. All animals survived a time span of 3 weeks, when electrodes were implanted into the ipsilateral $\mathrm{CA} 3$ region. While spontaneous focal seizures were recorded from the CA3 region of all WT mice (Figure 6A), indicating epileptogenesis, spontaneous seizures were completely absent in $f b$-Adk-def and $f b$-Adk-def/DPCPX mice (Figure 6, B and C), indicating that elevated

\section{Figure 7}

Representative EEGs recorded from the ipsilateral CA3 3 weeks after cell transplantation into KA-injected mice. (A) KA-injected animal treated with a sham procedure. (B) KA-injected recipient of WT control cells. (C) KA-injected recipient of adenosine-releasing $A d k^{-1-}$ ES cell-derived NPs. Scale bar: $2 \mathrm{~s}$.

forebrain adenosine prevents the development of spontaneous recurrent seizures. After completion of EEG recordings, all animals were sacrificed for histology. Analysis of cresyl violet-stained brain sections showed the presence of the ipsilateral CA3 lesion in WT (Figure $6 \mathrm{~A}$ ) and $f b$-Adk-def/DPCPX mice (Figure 6C) but not in $f b$-Adk-def mice (Figure 6B), indicating the presence of the epileptogenesis-initiating primary injury (Figure 6, B and H). Since astrogliosis and overexpression of endogenous ADK were linked to epileptogenesis (Figure 1), we analyzed expression of the astrocyte markers GFAP and ADK in the hippocampal formation in more detail. Whereas WT animals displayed a striking and highly localized astrogliotic response within the ipsilateral CA3 with colocalized overexpression of GFAP and ADK (Figure 6A), no signs of astrogliosis or upregulation of ADK were found in $f b$-Adk-def or $f b$-Adk-def/DPCPX mice, even though the latter showed SE-induced CA3 cell loss similar to that of WT (Figure 6, $\mathrm{B}$ and $\mathrm{C}$ ). The lack of astrogliosis, and consequently the absence of upregulated $\mathrm{ADK}$, resulting in the lack of spontaneous seizures in $f b$-ADK-def mice, demonstrate that elevated forebrain adenosine can prevent epileptogenesis. To our knowledge, this is the first demonstration of a transgenic animal with resistance to epileptogenesis.

Adk ${ }^{-/-}$ES cell-derived neural progenitor grafts prevent development of spontaneous seizures. To determine whether adenosine-releasing intrahippocampal stem cell-derived implants could prevent the development of epileptogenesis, 3 groups of mice received implants of adenosine-releasing $A d k^{-/-}$(KA-KO mice) (27) or respective WT (KA-WT mice) ES cell-derived neural precursor cells (NPs) or corresponding sham treatments (KA-SHAM mice) 24 hours after intraamygdaloid KA injection ( $n=6$ per group), i.e., at a time point at which the initial acute CA3 injury was already present. All animals survived a time span of 3 weeks, when electrodes were implanted into the ipsilateral CA3 region as described above. Spontaneous focal seizures (Figure 7, A and B) were recorded from the CA3 region of all KA-SHAM and KA-WT mice, with $4.2 \pm 1.4$ and $4.0 \pm 1.3$ seizures per hour and average durations of $17.3 \pm 5.9$ and $16.8 \pm 5.5$ seconds, respectively, values comparable to seizure parameters in KA-injected control mice (Table 2; $P>0.05$ ). In contrast, not a single spontaneous seizure was detected after recording KA-KO mice with $A d k^{-/-}$NP implants $(n=6)$ for 8 hours each (Figure $7 \mathrm{C}$ ), a time span equivalent to $>192$ seizures in a corresponding number of control animals $(n=6)$.

\section{Table 2}

Spontaneous electroencephalographic seizures recorded from the CA3 region

\begin{tabular}{|c|c|c|c|c|c|}
\hline Parameter & $A d k-T g(n=4)$ & KA-control $(n=6)$ & KA-SHAM $(n=6)$ & $\mathrm{KA-WT}(n=6)$ & $\mathrm{KA-KO}(n=6)$ \\
\hline Average duration (s) & $26.7 \pm 13.2$ & $17.5 \pm 5.8$ & $17.3 \pm 5.9$ & $16.8 \pm 5.5$ & 0 \\
\hline Frequency $(/ \mathrm{h})$ & $4.8 \pm 1.5$ & $4.3 \pm 1.5$ & $4.2 \pm 1.4$ & $4.0 \pm 1.3$ & 0 \\
\hline
\end{tabular}



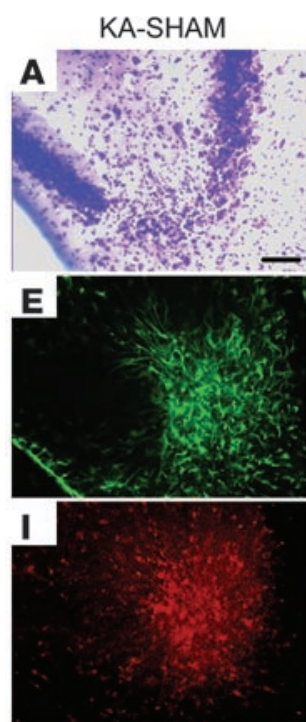

M
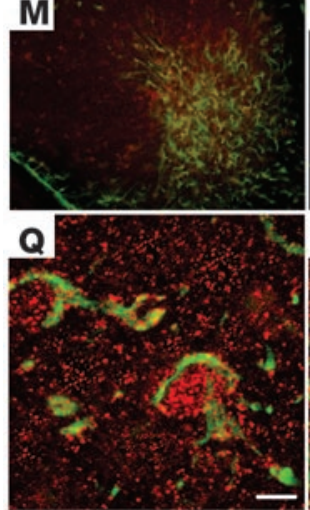

KA-WT
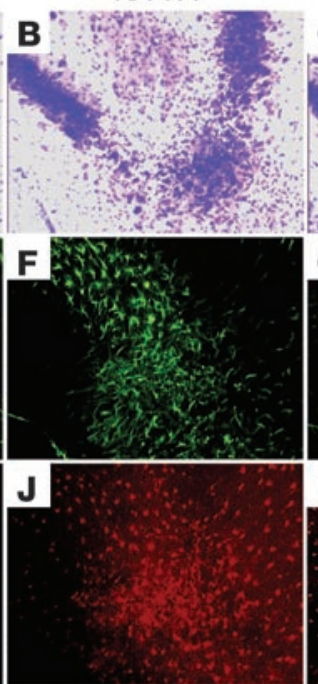

N
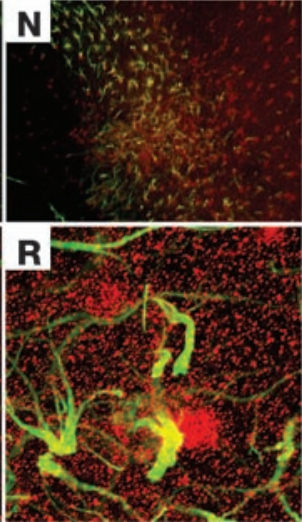

KA-KO

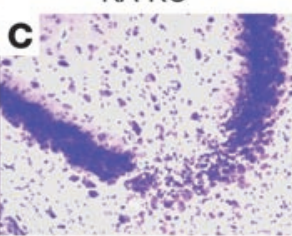

G

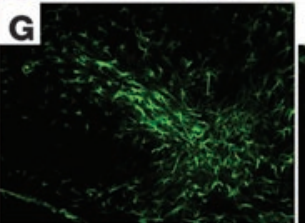

K

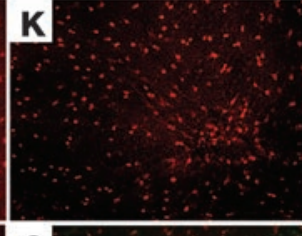

o
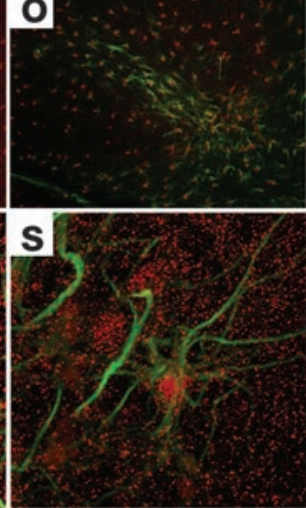
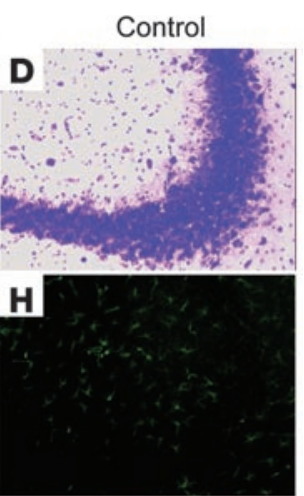

L

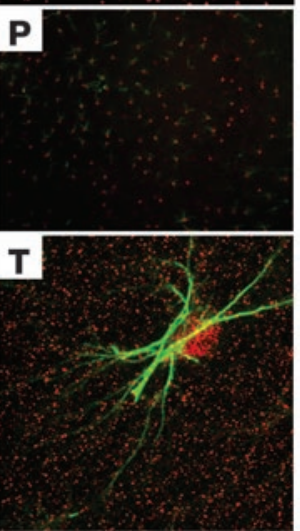

\section{Figure 8}

Histological assessment of the antiepileptogenic effect of $\mathrm{Adk}^{-1-} \mathrm{ES}$ cell-derived NPs assessed on coronal brain sections of mice 3 weeks after KA injection/cell transplantation. First column: Sham-treated KA-injected control animals. Second column: KA-treated recipients of WT cells. Third column: KA-treated recipients of $\mathrm{Adk}^{-1-}$ ES cellderived NPs. Fourth column: untreated control animals. (A-D) Cresyl violet staining demonstrating $\mathrm{CA} 3$ selective neuronal cell loss in all KA-injected animals. (E-H) GFAP immunofluorescence showing prominent CA3 gliosis in shamtreated and WT KA-injected animals, reduced gliosis in $\mathrm{Adk}^{-1-} \mathrm{NP}$ recipients, and lack of gliosis in untreated controls. (I-L) ADK immunofluorescence showing prominent upregulation of ADK in the KA-injected control animals but lack of ADK upregulation in $\mathrm{Adk}^{-1-} \mathrm{NP}$ recipients. (M-P) Overlay of GFAP and ADK immunofluorescence. (Q-T) Confocal analysis of GFAP and ADK immunofluorescence showing overexpression and redistribution of ADK (red) in the context of gliosis (green) in the KA-injected sham-treated and WT controls but not in the Adk ${ }^{-1-}$ NP recipients. For comparison, an astrocyte from the stratum radiatum of an untreated control animal is shown $(\mathbf{T})$, since no astrocytes are normally located within the CA3. Scale bars: $75 \mu \mathrm{m}(\mathbf{A}-\mathbf{P}) ; 10 \mu \mathrm{m}(\mathbf{Q}-\mathbf{T})$.
$A d k^{-/}$ES cell-derived neural progenitor grafts reduce astrogliosis and upregulation of $A D K$. After completion of the EEG recordings, i.e., 3 weeks after KA injection, all animals were processed for histochemistry. To verify the existence of the acute epileptogenesis triggering brain injury as becoming evident 24 hours after KA, i.e., before cell transplantation, we subjected coronal brain sections from KA-SHAM, KA-WT, or KA-KO animals and naive control animals (no KA injection) to cresyl violet staining. CA3-selective cell loss was only evident in the KA-injected animals (Figure 8, A-C), while the $\mathrm{CA} 3$ region remained intact in the control animals (Figure 8D). To investigate whether $A d k^{-/-}$NPs prevented spontaneous seizures via reduction of astrogliosis and reduced upregulation of ADK, we analyzed expression of the astrocyte markers GFAP and ADK in the hippocampal formation in the 4 groups of mice. KA-WT and KA-SHAM animals displayed a highly localized astrogliotic response within the ipsilateral CA3 (Figure 8, E and F), with colocalized overexpression of GFAP and ADK (Figure 8, M, N, Q, and $\mathrm{R}$ ). In contrast, astrogliosis was markedly reduced (Figure 8, G, $\mathrm{O}$, and $\mathrm{S}$ ) and no obvious upregulation of ADK became evident within the ipsilateral $\mathrm{CA} 3$ of the $\mathrm{KA}-\mathrm{KO}$ group representing the $A d k^{-/-}$graft recipients (Figure $8, \mathrm{~K}, \mathrm{O}$, and $\mathrm{S}$ ).

To generate quantitative data on GFAP and ADK expression, we determined the density of ADK immunoreactivity within the CA3 field and counted the number of GFAP-positive cells in ipsilateral and contralateral CA3 fields of KA-injected control animals and in ipsilateral hippocampus of KA-SHAM, KA-WT, and KA-KO animals (Figure 9). ANOVA analysis revealed a significant increase in the density of ADK immunoreactivity as well as the number of GFAP-positive astrocytes $(P<0.01$ and $P<0.001$, respectively) within the ipsilateral CA3 of the KA control animals (KA-SHAM and KA-WT). In contrast, the number of GFAP-positive astrocytes in the KA-KO animals was only moderately increased $(P<0.01)$, while changes in ADK immunoreactivity densities failed to attain statistical significance $(P>0.05)$. These data demonstrate that $A d k^{-/-} \mathrm{NP}-$ derived brain implants prevent the upregulation of host ADK and thus eliminate a major factor contributing to seizure generation.

Adk ${ }^{-/}$ES cell-derived neural progenitor cells display intrabippocampal location. In coronal brain sections from animals sacrificed 3 weeks after grafting, we detected dense cellular transplants in the vicinity of the surgical injection tract, located in the infrahippocampal cleft according to our previous studies (23) (Figure 10, A and B). Likewise, in all animals, we detected individual graft-derived cells in the CA1 region of the ipsilateral hippocampus (Figure 10, C and D). Most of these graft-derived cells showed a morphology and orientation comparable with mature hippocampal neurons, including an elaborate dendritic tree and a principal axon. We did not find any differences in graft location between WT and $A d k^{-/-}$graft recipients. In all animals $(n=12)$, the location of the graft was confined to the ipsilateral hippocampus, while the contralateral hippocampus as well as all other brain areas analyzed were devoid of graft-derived cells. 

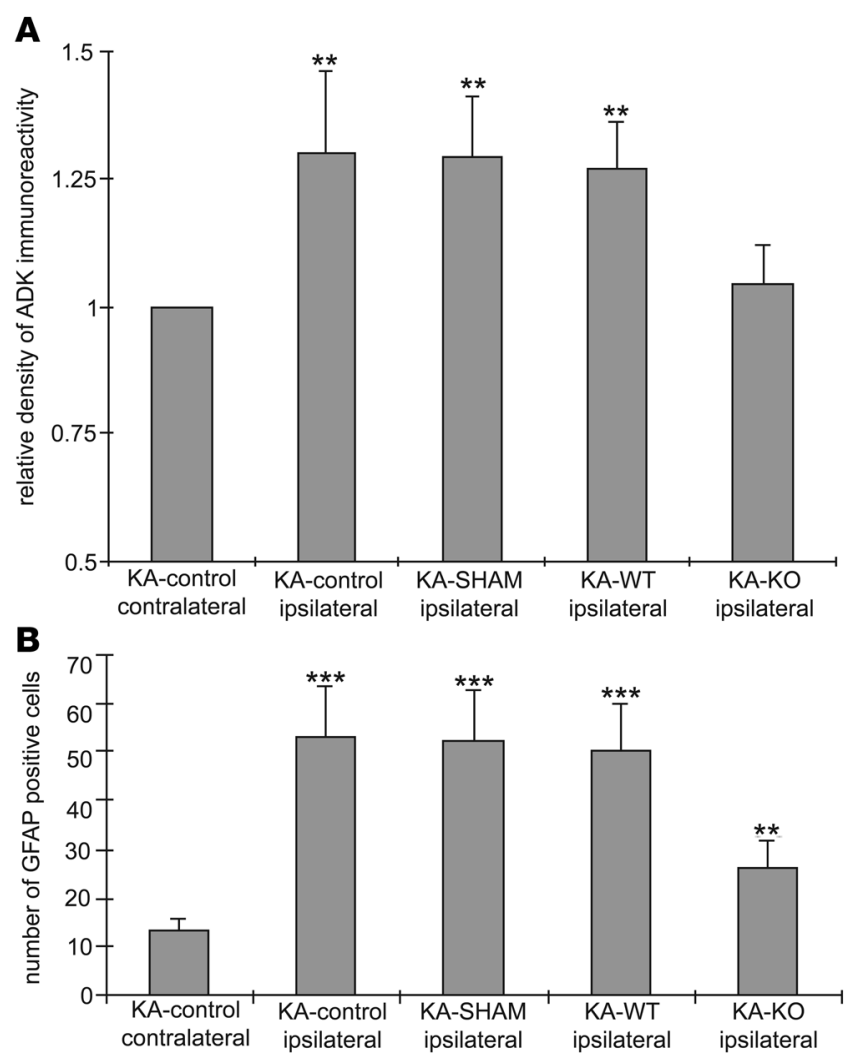

\section{Discussion}

As a long-term consequence of brain injury, astrogliosis is considered to be a hallmark of the epileptic brain. Autopsy and surgical resection specimens have demonstrated that chronic temporal lobe epilepsy and posttraumatic seizures may originate from gliotic scars $(28,29)$. The involvement of astrocytes in the pathogenesis of epileptogenesis can no longer be ignored since recent findings that astrocytes control synaptic transmission by the vesicular release of glutamate, the NMDA receptor coagonist D-serine, and ATP as a source of synaptic adenosine (2).

As a response to astrogliosis and chronic seizures, the adenosine system undergoes changes, which can promote further seizures. Thus downregulation of $\mathrm{A}_{1} \mathrm{Rs}$ has been described as a consequence of chronic seizures $(30,31)$. Likewise, a combined decrease in the density of $\mathrm{A}_{1} \mathrm{Rs}$ and metabolic changes leading to lower levels of adenosine were described in the hippocampus of epileptic rats (32). Indeed, overexpression of ADK within an epileptic hippocampus was associated with astrogliosis and focal seizures (18). Likewise, deficits in adenosinergic neuromodulation promote seizures in hippocampal slices $(10,33)$. Here we propose the ADK hypothesis of epileptogenesis and provide experimental evidence on 4 different levels:

Mouse model of focal epileptogenesis. We previously suggested that upregulation of ADK during astrogliosis might cause spontaneous seizures $(18,19)$. However, these studies were based on intrahippocampal injection of KA causing widespread hippocampal astrogliosis and upregulation of ADK. In this model of TLE, it was not possible to distinguish direct effects on hippocampal integrity caused by the excitotoxin kainate or secondary effects caused by the resulting SE. Widespread astrogliosis and upregulation of ADK in this model precluded the delineation of seizure generation at high spatial resolution. In the present investigation we therefore decided to study the

\section{Figure 9}

Quantitative assessment of ADK immunoreactivity and GFAP expression in the CA3 region. Quantitative data were generated from KA-control, KA-SHAM, KA-WT, and KA-KO animals 3 weeks after KA injection/cell transplantation. (A) The relative density of ADK immunoreactivity was determined on $\mathrm{DAB}$-stained sections by scanning $\mathrm{CA} 3 \mathrm{a}$ fields of $300 \times 200 \mu \mathrm{m}$ on 2 sections each from $n=6$ animals per treatment group. Data were normalized to the contralateral hippocampus of KA-injected control animals. (B) The total number of GFAP-positive cells in corresponding CA3a fields of $300 \times 200 \mu \mathrm{m}$ was determined by counting GFAP-positive cells on 2 sections each from $n=6$ animals per treatment group. Data analysis was done by ANOVA; mean \pm SD. ${ }^{* *} P<0.01 ;{ }^{* *} P<0.001$.

adenosine system in a model of focal epileptogenesis, in which we used unilateral intraamygdaloid injections of KA. This model has the advantage of inducing an acute injury restricted to the ipsilateral hippocampus without direct manipulation of the hippocampus (24). We believe that the chronic epileptogenic response to an acute intraamygdaloid KA-induced hippocampal injury as reported in this study (Figure 1) is a novel finding and involves a precipitating event (i.e., KA-induced SE), injury-induced cell death, highly localized astrogliosis, upregulation of glial-based ADK, and the development of focal spontaneous seizures at a frequency of $4.3 \pm 1.5$ seizures per hour. Remarkably, spontaneous seizures develop exclusively within the astrogliotic CA3 region but not elsewhere within the hippocampus; likewise, neither generalized seizures nor any convulsive seizures were detected. Since seizure recordings were performed during 8 hours of continuous monitoring in each of 6 animals, a total of $>200$ spontaneous seizures were recorded. However, the limitation to 8 hours of recording does not allow us to draw any conclusions on possible day/night fluctuations of seizure activity.

It is important to note that seizures originate in a focal area, with prominent neuronal cell death (Figure 1D). Nevertheless, neuronal processes originating from adjoining hippocampal areas
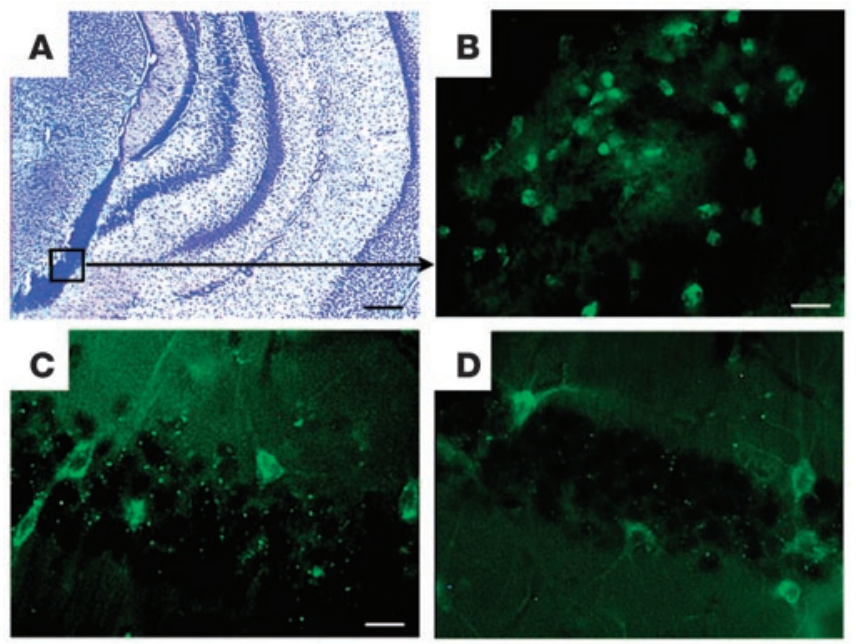

\section{Figure 10}

Adk ${ }^{-1}$ NP-derived cell implant 3 weeks after grafting. (A) Cresyl violetstained overview showing a dense cluster of implanted cells within the infrahippocampal cleft (box). (B) Green fluorescence staining of graftderived cells within the infrahippocampal cleft. (C and D) Graft-derived cells located within the ipsilateral CA1 region and displaying neuron-like morphology. Scale bars: $150 \mu \mathrm{m}$ (A); $20 \mu \mathrm{m}$ (B); $10 \mu \mathrm{m}$ (C and D). 
penetrate the injured CA3 (Figure $1 \mathrm{H}$ ), are present within an area of upregulated ADK (Figure 1L), and are likely the source for the highly localized CA3 seizures (Figure 1). The restriction of both upregulated $\mathrm{ADK}$ and focal seizures to the injured $\mathrm{CA} 3$ suggests the existence of an endogenous mechanism preventing the spread of seizures into regions with normal ADK expression. This endogenous protective mechanism may rely on the release of adenosine during seizures and the activation of $\mathrm{A}_{1} \mathrm{Rs}$, which have previously been implicated in keeping an epileptic focus localized $(34,35)$. These findings suggest that astrogliotic upregulation of ADK is an integral part of epileptogenesis and a cause for seizures. Indeed, previous studies in the mouse intrahippocampal KA model suggest that upregulation of ADK (first observed around 1 week after SE) precedes the development of spontaneous seizures (first observed around 2 weeks in the intrahippocampal KA model and around 12 days in this study) $(18,36)$. The results presented here document focal epileptogenesis at high spatial resolution and demonstrate that focal upregulation of ADK, which is not expected to alter the basal hippocampal adenosine-tone in other subregions, is sufficient to create a highly focused core of hyperexcitability.

Transgenic overexpression of ADK causes seizures. Adk-Tg mice display a striking overexpression of ADK in the hippocampal CA3 region (Figure 3D); however, in contrast to upregulated ADK as a component of epileptogenesis (Figure 1), transgenic overexpression is not associated with astrogliosis. Our finding of spontaneous seizures ( $4.8 \pm 1.5$ seizures per hour) in the CA3 of $A d k-T g$ mice at a similar frequency to seizures found in the KA-model 3 weeks after SE $(4.3 \pm 1.5$ seizures per hour) suggests that overexpression of ADK in the absence of astrogliosis is sufficient to cause spontaneous seizures.

Reduction of ADK prevents epileptogenesis. By comparing the causal sequence of events triggered by the intraamygdaloid application of KA in Adk transgenic mice, which either overexpress ADK (Adk-Tg) or have reduced ADK $(f b$-Adk-def) in their forebrains (Figure 3), we demonstrate that the severity of KA-induced SE (Figure 4) and the degree of SE-induced cell death (Figure 5) are inversely related to the levels of ADK expression. While WT mice developed typical type IV seizures and a well-defined lesion in the ipsilateral CA3 of the hippocampus, this strong seizure response and selective CA3 damage were never observed in the $f b$-Adk-def mice. Most importantly, in $f b$-Adk-def mice pretreated with the $\mathrm{A}_{1} \mathrm{R}$ antagonist DPCPX, the initial KA-induced brain injury was comparable with WT mice, but due to elevated forebrain adenosine, subsequent epileptogenesis was prevented (Figure 6C). The efficacy of DPCPX in restoring WT-like brain injury in $f b$-Adk-def mice suggests that $\mathrm{A}_{1}$ Rs are fully functional in $f b$-Adk-def mice, making secondary effects due to potential receptor desensitization unlikely. This DPCPX control experiment also demonstrates that $f b$-Adk-def mice are principally able to express KA-induced seizures, indicating full functionality of KA receptors in these animals. It is important to note that $f b$-Adk-def mice do not lack forebrain ADK. Such a complete lack of ADK would likely be lethal. Rather, these mutants are characterized by a subtle reduction (about 40\%) and altered cellular distribution of ADK compared with WT. Similarly, ADK is only increased 1.5-fold in Adk-Tg mice, an increase that is completely sufficient to enhance brain vulnerability. The importance of subtle changes in ADK activity makes sense from a biochemical point of view. Adenosine levels are mainly regulated by an active substrate cycle between adenosine and AMP involving ADK and 5'-nucleotidase. Thus small changes in ADK can rapidly translate into major changes in adenosine (37-39). Because of difficulties in performing correct local adenosine measurements in living brain tissue and because of large variations according to the methods used (40), we previously chose an indirect electrophysiological approach to demonstrate ADK-dependent changes in ambient adenosine, confirming a reduction of endogenous adenosine in the brain of $A d k-T g$ mice (19). In line with reduced levels of brain adenosine, Adk-Tg mice have increased susceptibility to ischemic cell death (20) and have cognitive deficits and altered sensitivity to psychomimetic drugs, which are compatible with reduced brain adenosine (41). Conversely, the reversal of adenosine-mediated neuroprotection in forebrains of $f b-A d k$-def mice by a dose of the $A_{1} R$ antagonist DPCPX, which does not influence the activity of normal adenosine in WT mice, indicates increased levels of adenosine in the forebrain of $f b$-Adk-def mice. Since levels of ATP are about 100,000-fold higher than those of adenosine (7), since adenosine is the only substrate for $\mathrm{ADK}$, and since the enzyme is not required for the de novo synthesis of purines, subtle changes in ADK are not likely to affect the pool of adenine nucleotides nor any other metabolites. The fact that rather modest changes in ADK are needed to produce major effects has obvious implications for future therapy.

Preventive cell therapy. Previous therapeutic approaches have demonstrated that focal brain implants of fibroblasts, myoblasts, and stem cells engineered to release adenosine are effective in the suppression of kindled seizures (22). However, these approaches share a common caveat with currently used therapeutic strategies to treat epilepsy, in affecting merely the symptoms of epilepsy (i.e., seizures) rather than providing a true cure. The findings reported here suggest that upregulation of ADK is a key component of epileptogenesis. Consequently, therapeutic augmentation of the adenosine system should prevent epileptogenesis. After triggering epileptogenesis in mice by intraamygdaloid KA, we transplanted $A d k^{-/-}$ES cell-derived NPs into the infrahippocampal cleft and demonstrate that these implants prevent epileptogenesis after acute brain injury. This becomes evident by the lack of spontaneous seizures 3 weeks after cell transplantation, by reduced astrogliosis, and most importantly, by lack of pathogenic upregulation of host ADK. To our knowledge, this is the first demonstration that epileptogenesis can be prevented therapeutically. Further studies are thus warranted to address issues of effective cell dosages, spatial requirements, and long-term effects of adenosine-releasing brain implants. Interestingly, the therapeutic efficacy of adenosine-releasing brain implants seems to depend on ADK expression levels. While the $A d k^{-/-}$ES cell-derived NPs used here (0\% ADK) provided complete protection from epileptogenesis, human mesenchymal stem cell implants with an RNAi-mediated knockdown of ADK $(20 \%-28 \%$ of residual ADK) provided only partial protection from acute SE-induced hippocampal injury (42). These findings may suggest a quantitative relation between ADK expression levels, cellular adenosine release, and functional outcome.

The link between expression levels of ADK and susceptibility to epileptogenesis described here allows defining high levels of ADK expression as a risk factor for epileptogenesis. This is of importance, since following an acute brain injury only a small number of patients will develop epilepsy. Thus identification of high ADK expression, e.g., by suitable PET ligands, would allow us to determine those patients at risk for developing epilepsy and would indicate initiation of adenosine-based preventive therapies.

The results presented here define ADK as an attractive target to prevent epileptogenesis. Indeed, ADK inhibitors, such as ABT-702 or related compounds, are effective in the suppression of established or induced seizures; since they can potentiate a site- and event-specific 
surge of endogenous adenosine, they have an improved side effect profile compared with $\mathrm{A}_{1} \mathrm{R}$ inhibitors $(43,44)$. However, the clinical development of another ADK inhibitor, GP-3966, was stalled due to the incidence of CNS hemorrhage in rats and dogs (45), and systemic inhibition of ADK may be toxic to the liver (26). Thus local or focal treatment approaches might be preferable to systemic pharmacotherapy. ADK-deficient brain implants, as demonstrated here, have the potential to locally restore the adenosinergic equilibrium within the brain, as well as to prevent pathogenetic upregulation of ADK and the emergence of spontaneous seizures during epileptogenesis. Thus focal cell-based augmentation of adenosine has been identified as a potentially novel principle to prevent epileptogenesis. In conclusion, the present study has identified ADK as a diagnostic marker to predict, and as a therapeutic target to prevent, epileptogenesis. Future research will determine which ADK-based treatment strategies would be most effective and compatible with clinical applications.

\section{Methods}

Generation offb-Adk-def mice. All animal procedures were performed in an AAALAC-accredited facility according to protocols approved by the Legacy Research IACUC and the principles outlined in the NIH Guide for the Care and Use of Laboratory Animals. Animals were kept in a 12-hour light/12-hour dark cycle, with lights on from 7 am to $7 \mathrm{pm}$ in individually ventilated isolator cages, and food and water were supplied ad libitum. Adktm 1-1- $: \operatorname{Tg}(U b i A d k): E m x 1-C r e-T g 3$ (fb-Adk-def) mice were generated by breeding Adktm 1-/-Tg(UbiAdk) (Adk-Tg) mice (19) with Emx1-Cre-Tg3 mice (25) on a C57BL/ 6 background (Figure $2 \mathrm{~B}$ ). The colony was maintained

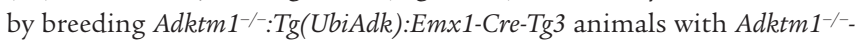
$\mathrm{Tg}$ (UbiAdk) mice, thus producing both genotypes as littermates and keeping the Adktm1 $1^{-1-}: \operatorname{Tg}(U b i A d k): E m x 1-C r e-T g 3$ mice heterozygous for the Cre allele and homozygous for the $\mathrm{Tg}(U b i A d k)$ allele.

Genotyping and immunohistochemical analysis. The endogenous Adk locus was analyzed in individual PCR reactions containing a mix of the primers o107, o108, and 0109 (27). The Adk transgene was identified by primers o141 and o142 (19). The combination of 0107 with 0108 gave rise to 640-bp products (WT Adk allele), whereas the combination of 0107 with 0109 gave rise to $840-b p$ products (Adk-KO allele). The combination of o141 and o142 gave rise to 420 -bp products, indicative for the transgene. PCR primers used for detection of Cre recombinase were o234, 5'-TGACAGCAATGCTGTTTCACTGG$3^{\prime}$, and o235, 5'-GCATGATCTCCGGTATTGAAACTCC-3'; the Cre-specific amplification product was $600 \mathrm{bp}$. The thermocycle of the PCR was 35 cycles at $95^{\circ} \mathrm{C}(15 \mathrm{~s}), 61^{\circ} \mathrm{C}(20 \mathrm{~s})$, and $70^{\circ} \mathrm{C}(90 \mathrm{~s})$. ADK immunohistochemistry was performed on coronal perfused brain sections from adult Adk-Tg, $f b$-Adk-def, and WT mice as previously described (17). For western blot analysis, brains from a different set of mice $(n=2$ per genotype) were divided into a sample containing forebrain and a sample containing brainstem and cerebellum. From these samples, crude homogenates were prepared and subjected to western blot analysis in triplicate, as previously described (19). ADK levels were normalized to a $\beta$-actin control and to WT ADK (set as 100\%).

Epileptogenesis model. Seizures were induced in adult male WT, Adk-Tg, and $f b$-Adk-def mice weighing $25-30 \mathrm{~g}$ by unilateral stereotaxic microinjection of $\mathrm{KA}$ into the basolateral amygdala nucleus based on stereotactic coordinates relative to bregma: AP, $-0.94 \mathrm{~mm}$; $\mathrm{ML},-2.85 \mathrm{~mm}$; and DV, $-3.75 \mathrm{~mm}$ (AP, anterior-posterior; $\mathrm{ML}$, medio-lateral; $\mathrm{DV}$, dorso-ventral) as previously described (5). Briefly, under anesthesia with $68.5 \% \mathrm{~N}_{2} \mathrm{O}, 30 \% \mathrm{O}_{2}$, and $1.5 \%$ isoflurane, mice were affixed with 3 recording electrodes (Plastics One Inc.) and a 26gauge steel guide cannula over the intact dura using dental cement. To perform the subsequent EEG recordings and drug injections in awake animals, the mice were placed into a plexiglass restrainer. Anesthesia was discontinued and EEG recordings commenced, and then a 31-gauge internal cannula was inserted into the lumen of the guide to inject $0.3 \mu \mathrm{g}$ KA in a volume of $0.2 \mu \mathrm{l}$ PBS, $\mathrm{pH} 7.4$, into the amygdala. The EEG was monitored for 30 min using a Nervus video-EEG recoding device until lorazepam $(6 \mathrm{mg} / \mathrm{kg})$ was administered i.v. to terminate seizures. The EEG was further monitored for up to $30 \mathrm{~min}$ to ensure seizure cessation. An observer unaware of the experimental treatment performed quantification of EEG records, and the duration of type IV seizure activity was calculated. Selected animals were injected once with $1 \mathrm{mg} / \mathrm{kg}$ i.p. 8-cyclopentyl-1,3-dipropylxanthine (DPCPX; Sigma-Aldrich) 30 min prior to intraamygdaloid KA injection. Three weeks after KA injection, a bipolar coated stainless steel electrode $(0.20 \mathrm{~mm}$ in diameter; Plastics One Inc.) was implanted into the ipsolateral $\mathrm{CA} 3$ region and fixed with a pedestal of dental acrylate. Coordinates for the CA3 electrodes (with toothbar at 0 ) were $2.18 \mathrm{~mm}$ caudal to bregma, $2.5 \mathrm{~mm}$ lateral to midline, and $2.25 \mathrm{~mm}$ ventral to dura. Additional animals received CA1 or cortical recording electrodes. Twenty-four hours after electrode implantation, each animal was subjected to one 8-hour session (from 10 am to $6 \mathrm{pm}$ ) of continuous EEG monitoring performed during the light period. An observer unaware of the experimental treatment performed quantification of EEG records.

Cell culture. WT and genetically altered ES cells ( $A d k^{+/+}$and $A d k^{-/-}$, respectively) used in these experiments have been described previously $(23,27)$. NPs were generated from $A d k^{-/-}$and $A d k^{+/+}$ES cells using a well-established stepwise differentiation protocol (46). NPs were routinely cultured on polyornithine-coated dishes in N3 medium as previously described (23). To keep the cells in a proliferative state, the growth factor FGF2 $(10 \mathrm{ng} / \mathrm{ml})$ was added daily. After neural differentiation, adenosine release from $\mathrm{Adk} \mathrm{k}^{-/-}$ cells amounted to $9.00 \pm 4.55 \mathrm{ng}$ adenosine $/ 10^{5}$ cells, while the amount of adenosine released from WT cells $\left(0.33 \pm 0.20 \mathrm{ng}\right.$ adenosine $/ 10^{5}$ cells $)$ was minimal (23). Prior to transplantation the cells were labeled with a commercial cell tracer kit (Vybrant CFDA SE Cell Tracer Kit; Invitrogen). The labeling efficiency was about $10 \%$.

Cell transplantation. All animals, including control animals, received daily immunosuppression with cyclosporine A $(15 \mathrm{mg} / \mathrm{kg}$, i.p.) initiated 2 days prior to cell transplantation, which was performed under anesthesia with $68.5 \% \mathrm{~N}_{2} \mathrm{O}, 30 \% \mathrm{O}_{2}$, and $1.5 \%$ isoflurane $24 \mathrm{~h}$ after $\mathrm{KA}$ injection. Immediately before transplantation, cells were harvested and resuspended at a concentration of 50,000 cells per $2.5 \mu \mathrm{l}$ in culture medium. Cell injections (50,000 cells per mouse) were performed using a glass capillary (inner diameter of tip, $70-90 \mu \mathrm{m}$ ). The cells were slowly injected in a volume of $2.5 \mu \mathrm{l}$ using a drill hole above the left hippocampus and a single diagonal injection tract spanning from coordinate $(\mathrm{AP},+1.6 ; \mathrm{ML},+1.2 ; \mathrm{DV}, 0.0)$ to coordinate (AP, $-2.8 ; \mathrm{ML},-1.75 ; \mathrm{DV},-4.0$ ), thus depositing the cells within the infrahippocampal cleft of the to-be-injured brain hemisphere as described previously (23). Cells were slowly injected $(1 \mu \mathrm{l} / \mathrm{min})$ while withdrawing the capillary $(1 \mathrm{~mm} / \mathrm{min})$. The capillary was fully retracted $5 \mathrm{~min}$ after injection to avoid reflux of cells. Using an identical procedure, shamtreated control animals $(n=6)$ received culture medium instead of cells.

Histology. Brains obtained $24 \mathrm{~h}$ following the KA injection were immediately frozen in 2 -methylbutane $\left(-30^{\circ} \mathrm{C}\right)$ and sectioned at $12 \mu \mathrm{m}$ on a cryostat. Coronal sections at the level of bregma $-1.7 \mathrm{~mm}$ were air dried, postfixed in $10 \%$ formalin (15 min), washed twice in PBS, and then processed for histopathology (cresyl violet staining) or for detection of DNA fragmentation (TUNEL) as previously described (5). The average number of TUNEL-positive cells per brain slice was quantified by counting TUNEL-positive cells from 3 adjacent brain sections, encompassing the entire CA1/3 field, derived from 3 animals from each genotype.

Three weeks after KA injection, the mice were transcardially perfused with $4 \%$ paraformaldehyde in phosphate buffer $(0.15 \mathrm{M}, \mathrm{pH} 7.4)$. The brains were postfixed in the same fixative at $4^{\circ} \mathrm{C}$ for $6 \mathrm{~h}$ and cryoprotected in $10 \%$ DMSO in PBS (vol/vol) before being cut into $40-\mu \mathrm{m}$ coronal sections. Six sections from each brain, representing different levels of the 
hippocampal formation encompassing the complete rostrocaudal extent of the hippocampus, were mounted onto gelatin-coated slides and stained with cresyl violet. For the immunohistochemical detection of GFAP and ADK, we followed our previously published procedures and stained sections adjacent to the cresyl violet-stained sections (17). Further adjacent sections were stained with NFM (Santa Cruz Biotechnology Inc.) and DAPI (Invitrogen). Cellular implants were identified by tracer-derived green fluorescence. GFAP and ADK expression was quantified by analyzing fields of $200 \times 300 \mu \mathrm{m}$ encompassing the entire CA3a region. Corresponding fields from 2 sections from each animal ( $n=6$ animals per group) were analyzed by scanning ADK immunofluorescence on DAB-stained slices using a Kodak imaging device and by counting individual GFAP-positive cells. Data were analyzed by ANOVA with Student-Newman-Keuls test.

1. Pitkanen, A., and Sutula, T.P. 2002. Is epilepsy a progressive disorder? Prospects for new therapeutic approaches in temporal-lobe epilepsy. Lancet Neurol. 1:173-181.

2. Haydon, P.G., and Carmignoto, G. 2006. Astrocyte control of synaptic transmission and neurovascular coupling. Physiol. Rev. 86:1009-1031.

3. Tian, G.F., et al. 2005. An astrocytic basis of epilepsy. Nat. Med. 11:973-981.

4. Arabadzisz, D., Antal, K., Parpan, F., Emri, Z., and Fritschy, J.M. 2005. Epileptogenesis and chronic seizures in a mouse model of temporal lobe epilepsy are associated with distinct EEG patterns and selective neurochemical alterations in the contralateral hippocampus. Exp. Neurol. 194:76-90.

5. Shinoda, S., et al. 2004. Bim regulation may determine hippocampal vulnerability after injurious seizures and in temporal lobe epilepsy. J. Clin. Invest. 113:1059-1068.

6. Shetty, A.K., Zaman, V., and Hattiangady, B. 2005. Repair of the injured adult hippocampus through graft-mediated modulation of the plasticity of the dentate gyrus in a rat model of temporal lobe epilepsy. J. Neurosci. 25:8391-8401.

7. Fredholm, B.B., Chen, J.F., Cunha, R.A., Svenningsson, P., and Vaugeois, J.M. 2005. Adenosine and brain function. Int. Rev. Neurobiol. 63:191-270.

8. Johansson, B., et al. 2001. Hyperalgesia, anxiety, and decreased hypoxic neuroprotection in mice lacking the adenosine A1 receptor. Proc. Natl. Acad. Sci. U. S. A. 98:9407-9412.

9. Huber, A., et al. 2001. Grafts of adenosine-releasing cells suppress seizures in kindling epilepsy. Proc. Natl. Acad. Sci. U. S. A. 98:7611-7616.

10. Etherington, L.A., and Frenguelli, B.G. 2004. Endogenous adenosine modulates epileptiform activity in rat hippocampus in a receptor subtype-dependent manner. Eur. J. Neurosci. 19:2539-2550.

11. Dunwiddie, T.V. 1999. Adenosine and suppression of seizures. Adv. Neurol. 79:1001-1010.

12. Dunwiddie, T.V. 1980. Endogenously released adenosine regulates excitability in the in vitro hippocampus. Epilepsia. 21:541-548.

13. Dragunow, M., Goddard, G.V., and Laverty, R. 1985. Is adenosine an endogenous anticonvulsant? Epilepsia. 26:480-487.

14. Lee, K.S., Schubert, P., and Heinemann, U. 1984. The anticonvulsive action of adenosine: a postsynaptic, dendritic action by a possible endogenous anticonvulsant. Brain Res. 321:160-164.

15. During, M.J., and Spencer, D.D. 1992. Adenosine: a potential mediator of seizure arrest and postictal refractoriness. Ann. Neurol. 32:618-624.

16. Boison, D. 2006. Adenosine kinase, epilepsy and stroke: mechanisms and therapies. Trends Pharma-

\section{Acknowledgments}

This project was supported by NIH grant NS047622-01, the Good Samaritan Hospital Foundation, and the Epilepsy Research Foundation through the generous support of Arlene \& Arnold Goldstein Family Foundation.

Received for publication August 27, 2007, and accepted in revised form October 31, 2007.

Address correspondence to: Detlev Boison, Legacy Research, R.S. Dow Neurobiology Laboratories, 1225 NE 2nd Avenue, Portland, Oregon 97232, USA. Phone: (503) 413-1754; Fax: (503) 413-5465; E-mail: dboison@downeurobiology.org.

col. Sci. 27:652-658.

17. Studer, F.E., et al. 2006. Shift of adenosine kinase expression from neurons to astrocytes during postnatal development suggests dual functionality of the enzyme. Neuroscience. 142:125-137.

18. Gouder, N., Scheurer, L., Fritschy, J.-M., and Boison, D. 2004. Overexpression of adenosine kinase in epileptic hippocampus contributes to epileptogenesis. J. Neurosici. 24:692-701.

19. Fedele, D.E., et al. 2005. Astrogliosis in epilepsy leads to overexpression of adenosine kinase resulting in seizure aggravation. Brain. 128:2383-2395.

20. Pignataro, G., Simon, R.P., and Boison, D. 2007. Transgenic overexpression of adenosine kinase aggravates cell death in ischemia. J. Cereb. Blood Flow Metab. 27:1-5.

21. Boison, D. 2005. Adenosine and epilepsy: from therapeutic rationale to new therapeutic strategies. Neuroscientist. 11:25-36.

22. Boison, D. 2007. Adenosine-based cell therapy approaches for pharmacoresistant epilepsies. Neurodegener. Dis. 4:28-33.

23. Li, T., et al. 2007. Suppression of kindling epileptogenesis by adenosine releasing stem cell-derived brain implants. Brain. 130:1276-1288.

24. Araki, T., Simon, R.P., Taki, W., Lan, J.Q., and Henshall, D.C. 2002. Characterization of neuronal death induced by focally evoked limbic seizures in the C57BL/6 mouse. J. Neurosci. Res. 69:614-621.

25. Iwasato, T., et al. 2004. Dorsal telencephalon-specific expression of Cre recombinase in PAC transgenic mice. Genesis. 38:130-138.

26. Boison, D., et al. 2002. Neonatal hepatic steatosis by disruption of the adenosine kinase gene. Proc. Natl. Acad. Sci. U. S. A. 99:6985-6990.

27. Fedele, D.E., et al. 2004. Engineering embryonic stem cell derived glia for adenosine delivery. Neurosci. Lett. 370:160-165.

28. Tashiro, A., Goldberg, J., and Yuste, R. 2002. Calcium oscillations in neocortical astrocytes under epileptiform conditions. J. Neurobiol. 50:45-55.

29. Duffy, S., and MacVicar, B.A. 1999. Modulation of neuronal excitability by astrocytes. Adv. Neurol. 79:573-581.

30. Rebola, N., et al. 2005. Long-term effect of convulsive behavior on the density of adenosine A1 and A $2 \mathrm{~A}$ receptors in the rat cerebral cortex. Epilepsia. 46(Suppl. 5):159-165.

31. Glass, M., et al. 1996. Loss of A1 adenosine receptors in human temporal lobe epilepsy. Brain Res. 710:56-68.

32. Rebola, N., et al. 2003. Decrease of adenosine $A_{1}$ receptor density and of adenosine neuromodulation in the hippocampus of kindled rats. Eur. J. Neurosci. 18:820-828.
33. Dulla, C.G., et al. 2005. Adenosine and ATP link $\mathrm{P}-\mathrm{CO}_{2}$ to cortical excitability via $\mathrm{pH}$. Neuron. 48:1011-1023.

34. Fedele, D.E., Li, T., Lan, J.Q., Fredholm, B.B., and Boison, D. 2006. Adenosine $A_{1}$ receptors are crucial in keeping an epileptic focus localized. Exp. Neurol. 200:184-190.

35. Kochanek, P.M., et al. 2006. Adenosine A1 receptor knockout mice develop lethal status epilepticus after experimental traumatic brain injury. J. Cereb. Blood Flow Metab. 26:565-575.

36. Riban, V., et al. 2002. Evolution of hippocampal epileptic activity during the development of hippocampal sclerosis in a mouse model of temporal lobe epilepsy. Neuroscience. 112:101-111.

37. Arch, J.R., and Newsholme, E.A. 1978. Activities and some properties of $5^{\prime}$-nucleotidase, adenosine kinase and adenosine deaminase in tissues from vertebrates and invertebrates in relation to the control of the concentration and the physiological role of adenosine. Biochem. J. 174:965-977.

38. Bontemps, F., Van den Berghe, G., and Hers, H.G. 1983. Evidence for a substrate cycle between AMP and adenosine in isolated hepatocytes. Proc. Natl. Acad. Sci. U. S. A. 80:2829-2833.

39. Bontemps, F., Vincent, M.F., and Van den Berge, G. 1993. Mechanisms of elevation of adenosine levels in anoxic hepatocytes. Biochem. J. 290:671-677.

40. Delaney, S.M., and Geiger, J.D. 1996. Brain regional levels of adenosine and adenosine nucleotides in rats killed by high-energy focused microwave irradiation. J. Neurosci. Methods. 64:151-156.

41. Yee, B.K., Singer, P., Chen, J.F., Feldon, J., and Boison, D. 2007. Transgenic overexpression of adenosine kinase in brain leads to multiple learning impairments and altered sensitivity to psychomimetic drugs. Eur. J. Neurosci. 26:3237-3252.

42. Ren, G., et al. 2007. Lentiviral RNAi-induced downregulation of adenosine kinase in human mesenchymal stem cell grafts: a novel perspective for seizure control. Exp. Neurol. 208:26-37.

43. Kowaluk, E.A., and Jarvis, M.F. 2000. Therapeutic potential of adenosine kinase inhibitors. Expert Opin. Investig. Drugs. 9:551-564.

44. McGaraughty, S., Cowart, M., Jarvis, M.F., and Berman, R.F. 2005. Anticonvulsant and antinociceptive actions of novel adenosine kinase inhibitors. Curr. Top. Med. Chem. 5:43-58.

45. McGaraughty, S., and Jarvis, M.F. 2006. Purinergic control of neuropathic pain. Drug Dev. Res. 67:376-388.

46. Okabe, S., Forsberg-Nilsson, K., Spiro, A.C., Segal, M., and McKay, R.D. 1996. Development of neuronal precursor cells and functional postmitotic neurons from embryonic stem cells in vitro. Mech. Dev. 59:89-102. 